\title{
A Construction of Pooling Designs with Some Happy Surprises
}

\author{
A. D’YACHKOV, ${ }^{1}$ FRANK HWANG ${ }^{2}$ ANTONY MACULA, ${ }^{1,3}$ PAVEL VILENKIN, ${ }^{1}$ and \\ CHIH-WEN WENG ${ }^{4}$
}

\begin{abstract}
The screening of data sets for "positive data objects" is essential to modern technology. A (group) test that indicates whether a positive data object is in a specific subset or pool of the dataset can greatly facilitate the identification of all the positive data objects. A collection of tested pools is called a pooling design. Pooling designs are standard experimental tools in many biotechnical applications. In this paper, we use the (linear) subspace relation coupled with the general concept of a "containment matrix" to construct pooling designs with surprisingly high degrees of error correction (detection.) Error-correcting pooling designs are important to biotechnical applications where error rates often are as high as $15 \%$. What is also surprising is that the rank of the pooling design containment matrix is independent of the number of positive data objects in the dataset.
\end{abstract}

Key words: pooling designs, error correction.

\section{INTRODUCTION}

$\mathbf{T}$

He SCREENING OF BIOLOGICAL SETS OF OBJECTS, e.g., blood samples, cells, clones, macromolecules, is an essential but often laborious aspect of modern biotechnology. In a few instances, the screening of large libraries, e.g., peptide, cDNA, monoclonal antibody, for a relatively few number of positive objects has become a routine experimental procedure. See Bruno et al. (1995). Similar approaches have also been proposed for contig sequencing (Grebinski and Kucherov, 1998), determination of exon boundaries in eukaryotic genes (Pevzner, 2000), detecting gene complex (Torney, 1999), micro-array quality control (Colburn et al., 2002), and disease gene mapping (Flodman et al., 2001).

Whenever the objective is to find "needles in a haystack," a test indicating whether at least one needle is in a specific part of the haystack can greatly facilitate the isolation of the "needles." Such tests are

\footnotetext{
${ }^{1}$ Department of Probability Theory, Faculty of Mechanics and Mathematics, Moscow State University, Moscow, 119992, Russia.

${ }^{2}$ Department of Applied Mathematics, National Chiao Tung University, 1001 Ta Hsueh Road, Hsinchu 30050 , Taiwan. This research was partially supported by a Republic of China NSC grant 92-2115-M-009-014.

${ }^{3}$ Department of Mathematics, College at Geneseo, State University of New York, Geneseo, NY 14454, USA. This research was partially supported by NSF-DMS 0107179.

${ }^{4}$ Department of Applied Mathematics, National Chiao Tung University, 1001 Ta Hsueh Road, Hsinchu 30050, Taiwan. This research was partially supported by a Republic of China NSC grant 91-2005-M-009-008.
} 
called binary group tests, and the general mathematical method behind the identification of the "needles" using such tests is called group testing (Farach et al., 1997). If we have a finite ground set or population containing elements that can be uniquely characterized as positive or negative, we refer to the collection of positive elements as the positive subset $P$. In the abstract group testing problem, $P$ must be identified by performing 0,1 tests on subsets of the population.

One applied aim is to consider screening situations where we have a biological set of objects containing a relatively small number of data points (e.g., clones) which have a measurable attribute or function that can characterize them as "positive." This subcollection is initially unknown to the experimenter, and it is the object of the search. A group of biological objects taken from a larger set of objects is called a pool. A pool assay is a 0,1 test to determine if at least one member of the pool is positive. The practical goal here is to determine a large portion of $P$ from the pool assays. The collection of pools taken from a biological set of objects is called a pooling design.

Much of the current effort of the Human Genome Project involves the screening of large recombinant DNA libraries to isolate clones containing a particular DNA sequence.... This screening is important for diseasegene mapping and also for large-scale clone mapping.... More generally, efficient screening techniques can facilitate a broad range of basic and applied biological research. (Bruno et al., 1995)

For example, using probes to screen DNA libraries of clones fits the group testing paradigm in the following way: The population is the DNA library which consists of thousands of separate recombinant DNA clones, each of which represents some contiguous piece of a contiguous superpiece of DNA. A unique, identifiable, predetermined, and contiguous DNA subpiece is called a sequenced tagged site (STS). A clone is called positive for an STS if it contains that STS. A pool is a subset of the clones that are mixed together and tested by exposing the entire group to a chemical probe. A pool is labeled positive for an STS if the probe chemically indicates its presence. In other words, if the tests are error-free, then a pool is labeled positive for an STS if and only if that pool contains at least one clone that contains that STS.

Generally, because bioinformatic applications are often automated, parallel rather than sequential screening methods are preferred. See Farach et al. (1997) for other screening cost factors. Long before the advent of bioinformatics, consideration of analogous factors in other testing, screening, or coding situations lead to the development of nonadaptive group testing. See Du and Hwang (2000). In NGT, one must decide exactly which pools to test before any testing occurs. A NGT algorithm is sometimes referred to as a onestage algorithm. A two-stage algorithm is a nearly nonadaptive algorithm. In a trivial two-stage algorithm, all nontrivial pools occur in the first stage. After the first stage is complete, one has a set the candidate positives. In the second stage, each candidate positive is individually tested to see whether it is an actual positive.

When screening biological sets, errors almost always occur during the testing procedure. This paper addresses a new class of pooling designs that can cope with large numbers of errors.

\section{2. d-DISJUNCT MATRICES AS NONADAPTIVE POOLING DESIGN MODELS}

We will use the terminology of clone library screening for convenience. Suppose there are $n$ clones including at most $d$ positive ones (others are negative). A (group) test is applicable to an arbitrary subset of clones with two possible outcomes: a negative outcome indicates all clones in the subset are negative, and a positive outcome indicates otherwise. A pooling design is a specification of all tests so that they can be performed simultaneously with the goal being to identify all positive clones with a small number of tests. A pooling design $M$ can be represented by a binary incidence matrix where the columns represent clones, the rows represent tests, and $m_{i j}=1$ if and only if clone $j$ is contained in the subset of test $i$.

Suppose $M$ has $t$ rows. Then the $t$ outcomes can also be represented by a $t$-vector $V=\left(v_{1}, \ldots, v_{t}\right)^{t}$, where $v_{i}=1$ if and only if the outcome of test $i$ is positive ( $v_{i}=0$ otherwise). Note that $V$ is the Boolean sum of the set of positive clones. Therefore, it is convenient to view a column vector $C$ as a subset $S$ of the base set $\{1,2, \ldots, t\}$, where $i \in S$ if and only if $C$ has an 1 -entry in row $i$. Then we can say that $V$ is the union of the set of positive clones.

$M$ is called $d$-disjunct if no union of any $d$ columns covers another column. A $d$-disjunct matrix not only identifies the up-to- $d$ positive clones, but it does so with a simple decoding. Namely, a clone is 
positive if and only if it (as a column) is contained by $V$. This is because a negative clone (column) has at least one row not covered by the union of the up-to- $d$ positive clones; such a row then has a negative outcome which identifies the clone as negative. The notion of $d$-disjunctness was first raised by Kautz and Singleton (1964) in the study of superimposed codes. It was also studied by Erdös, Frankl, and Füredi (1985) under the name of $d$-cover-free family in extremal set theory. The $d$-disjunct matrices have become the most important tool in the construction of deterministic pooling designs. Although many constructions have been proposed, the existence of $d$-disjunct matrices is still sparse.

Macula (1996a) proposed a novel way of constructing $d$-disjunct matrices which uses the containment relation in a structure. More specifically, let $[m]:=\{1,2, \ldots, m\}$ be the base set. Then each of the $n$ columns is labeled by a (distinct) $k$ subset of $[m]$, assuming $n \leq\left(\begin{array}{c}m \\ k\end{array}\right)$, and each of the $\left(\begin{array}{c}m \\ d\end{array}\right)$ rows is labeled by a (distinct) $d$-subset of $[m]$, where $d<k<m ; m_{i j}=1$ if and only if the label of row $i$ is contained in the label of column $j$. He proved that $M$ is $d$-disjunct.

Huang and Weng (2004) generalized the construction to an arbitrary atomic semi-lattice where the elements can be ranked. Again, label the columns by a subset of the rank $k$ elements, and label the rows by all rank $d$ elements, $d<k$, and then $M$ is $d$-disjunct.

Ngo and Du (2002) further extended the construction to some geometric structures, such as simplicial complexes, and some graph properties, such as matchings. It is safe to say the "containment matrix" method has opened a new door for constructing $d$-disjunct matrices from many mathematical structures. However, the basic result in all these constructions is invariably that, to obtain a $d$-disjunct matrix, use all rank $d$ elements for rows.

One practical problem with this type of construction is that a large $n$ forces $d$ to be large. Then the number of tests could be too large as there are too many rank $d$ elements. This led Macula (1999) to propose using the rank 2 elements for rows, regardless of the real $d$. He showed that while there is no guarantee all positive clones will be identified, the probability of success is still satisfactory when $d$ does not deviate too much from 2. Ngo and Du made a similar comment.

In this paper, we show that the containment matrix which uses rank $r$ of elements for rows has the degree $d$ of disjunctness, where $r$ can be much less than $d$. In fact, $r$ can be any number from 1 to $k-1$ ( $k$ is the lever for columns), while $d \leq q^{r}$ for some constant $q$. This is the first happy surprise. Since we can choose $r=1$, we also have better control on the number of tests.

\section{THE ERROR-CORRECTING CAPABILITY}

Biological experiments are notorious for producing erroneous outcomes. Therefore, it would be wise for pooling designs to allow some outcomes to be affected by errors. Macula (1996b) proposed the notion of $d^{e}$-disjunct to reflect the error-correcting capability of a $d$-disjunct matrix. A $d$-disjunct matrix is $d^{e}$-disjunct if a column has at least $e+11$-entries not covered by the union of any other $d$ columns. The $d^{0}$-disjunct would then be the regular $d$-disjunct.

In Hwang (2003) it was misclaimed that a $d^{e}$-disjunct matrix can correct $e$ errors. The argument was that if we try all subsets $E$ of up to $e$ elements as the candidate set of errors and adjust the outcome set $V$ to $V \cup E$, then when $E$ is the true error set, a positive clone $C$ must be contained in $V \cup E$. On the other hand, a negative clone $C$ has at least $e+11$-entries not covered by the set of up to $d$ positive clones; i.e., $C$ has at least $e+1$ negative outcomes. At most $e$ of them can be converted to positive by errors; thus, at least one negative outcome is not covered by $V$. The problem of this argument is that we need to show that $C$ has at least one negative outcome not covered by $V \cup E$. The following is a counterexample.

Example 3.1. $d=2, e=1$. Column 1 is the only positive clone while $v_{3}$ is an error.

$$
M=\left(\begin{array}{lll}
1 & 0 & 0 \\
1 & 0 & 0 \\
0 & 1 & 0 \\
0 & 1 & 0 \\
0 & 0 & 1 \\
0 & 0 & 1
\end{array}\right) \quad V=\left(\begin{array}{l}
1 \\
1 \\
1 \\
0 \\
0 \\
0
\end{array}\right)
$$

When $E=\{4\}, V \cup E=(1,1,1,1,0,0)^{t}$ covers column 1 and 2 . 
Thus, the correct version should be te following.

Theorem 3.2. $A d^{2 e}$-disjunct matrix is e-error-correcting.

Proof. For a positive clone $C$, the argument is as before that there exists a candidate set $E$ such that $C \subseteq V \cup E$.

A negative clone $C$ has at least $2 e+1$ 1-entries not covered by the set $D$ of up to $d$ positive clones, hence, at least $2 e+1$ negative outcomes. The $e$ of them may be converted to positive by errors and another $e$ of them by $E$, but at least one negative outcome is not covered by $V \cup E$.

For the reason that a $d^{e}$-disjunct matrix is not really $e$-correcting, and also that $d^{0}$-disjunct $=d$-disjunct is not really customary, we propose to use the term $d^{z}$-disjunct while $z$ is the minimum (over $C$ ) number of 1-entries in $C$ not covered by the union of any other $d$ columns. Theorem 3.2 then can be restated as the following.

Theorem 3.3. A $d^{z}$-disjunct matrix can detect $z-1$ errors and correct $\left\lfloor\frac{z-1}{2}\right\rfloor$ errors.

In particular, a $d$-disjunct matrix has no error-tolerance.

If an extra round of confirmatory tests is allowed, then a $d^{z}$-disjunct matrix can indeed correct $z-1$ errors. First, we need a lemma. Let $H(X, Y)$ denote the Hamming distance between two binary vectors $X, Y$ of the same length.

Lemma 3.4. Let $M$ be a $d^{z}$-disjunct matrix and let $S_{1} S_{2}$ be two distinct subsets of columns with $\left|S_{1}\right| \leq d,\left|S_{2}\right| \leq d$. Let $U_{i}$ be the union of the set $S_{i}$ for each $i=1,2$. Then

1. $H\left(U_{1}, U_{2}\right) \geq z$ if either $S_{1} \subseteq S_{2}$ or $S_{2} \subseteq S_{1}$;

2. $H\left(U_{1}, U_{2}\right) \geq 2 z$ if otherwise.

Proof. These are trivial by using the $d^{z}$-disjunct property.

Theorem 3.5. A $d^{z}$-disjunct matrix corrects $z-1$ errors with an extra round of at most $d$ confirmatory tests.

Proof. Take all subsets $S$ of columns of $M$ with $|S| \leq d$ and $H(U, V) \leq z-1$, where $U$ is the union of $S$. Let $S_{1}, S_{2}$ be two such sets. The $H\left(U_{1}, U_{2}\right) \leq 2(z-1)<2 z$. By Lemma 3.4, either $S_{1} \subseteq S_{2}$ or $S_{2} \subseteq S_{1}$. Therefore, the set $\{S\}$ is a chain. Hence, $\{S\}$ has at most $d$ members. Since $H(D, V) \leq z-1$, $D \in\{S\}, D$ can be identified by testing at most $d$ columns in the maximal chain of $\{S\}$.

Not many constructions of $d^{z}$-disjunct matrices have been known. Macula (1996b), and also see Hwang (2003), gave a construction for $d^{4}$, and recently Ngo and Du gave a construction for $d^{d+1}$. We will show that the construction delivering the first happy surprise mentioned in Section 1 not only yields $d$-disjunct matrices, but also $d^{z}$-disjunct matrices with the $z$-value much greater than 4 or $d+1$. This is the second happy surprise.

\section{THE CONSTRUCTION}

Consider the $m$-dimensional space, or simply $m$-space, of $G F(q)$ where $q$ is a prime or a prime power. Let $\left[\begin{array}{c}m \\ k\end{array}\right]_{q}$ denote the number of $k$-dimensional subspaces, or simply $k$-space. It is well known (van Lint and Wilson, 1992, p. 291) that the following is true. 
Lemma 4.1.

$$
\left[\begin{array}{c}
m \\
k
\end{array}\right]_{q}=\frac{\left(q^{m}-1\right)\left(q^{m-1}-1\right) \cdots\left(q^{m-k+1}-1\right)}{\left(q^{k}-1\right)\left(q^{k-1}-1\right) \cdots(q-1)}
$$

and

$$
\left[\begin{array}{c}
m \\
k
\end{array}\right]_{q}=\left[\begin{array}{c}
m \\
m-k
\end{array}\right]_{q}
$$

Definition 4.2. Fix integers $1 \leq r<k<m$. Let $M(m, k, r)$ be the 01-matrix by taking all $k$-spaces (from an underlying $m$-space) as columns and all $r$-spaces as rows. $M(m, k, r)$ has $a 1$ in row $i$ and column $j$ if and only if $i$ is contained in $j$.

$M(m, k, r)$ was first studied by Yakir (1993) from a linear algebra point of view and by Ngo and Du (2002) from a pooling design point of view. $M(m, k, r)$ is easily checked to be a ranked atomic semi-lattice, thus the matrix is $r$-disjunct and hence (Huang and Weng, 2004) $d^{z}$-disjunct for some $1 \leq d \leq r$ and

$$
z=\left[\begin{array}{l}
k-d \\
r-d
\end{array}\right]_{q}
$$

Note that the construction still requires the row rank $r$ being at least as large as the upper bound $d$ of the number of positive clones. We now show that $r$ can be much less than $d$. First, we give a lemma.

\section{Lemma 4.3.}

$$
\left[\begin{array}{l}
k \\
r
\end{array}\right]_{q}-\left[\begin{array}{c}
k-1 \\
r
\end{array}\right]_{q}=q^{k-r}\left[\begin{array}{l}
k-1 \\
r-1
\end{array}\right]_{q} \quad(0 \leq r<k)
$$

Proof.

$$
\begin{aligned}
{\left[\begin{array}{l}
k \\
r
\end{array}\right]_{q} } & -\left[\begin{array}{c}
k-1 \\
r
\end{array}\right]_{q} \\
& =\frac{\left(q^{k}-1\right)\left(q^{k-1}-1\right) \cdots\left(q^{k-r+1}-1\right)}{\left(q^{r}-1\right)\left(q^{r-1}-1\right) \cdots(q-1)}-\frac{\left(q^{k-1}-1\right)\left(q^{k-2}-1\right) \cdots\left(q^{k-r}-1\right)}{\left(q^{r}-1\right)\left(q^{r-1}-1\right) \cdots(q-1)} \\
& =\frac{\left(q^{k}-1\right)-\left(q^{k-r}-1\right)}{q^{r}-1} \cdot \frac{\left(q^{k-1}-1\right) \cdots\left(q^{k-r+1}-1\right)}{\left(q^{r-1}-1\right) \cdots(q-1)} \\
& =q^{k-r}\left[\begin{array}{c}
k-1 \\
r-1
\end{array}\right]_{q} .
\end{aligned}
$$

Theorem 4.4. Suppose $k-r \geq 2$ and set $p:=\frac{q\left(q^{k-1}-1\right)}{q^{k-r}-1}$. Then $M(m, k, r)$ is $d^{z}$-disjunct for $1 \leq d \leq p$ and

$$
z=q^{k-r}\left[\begin{array}{l}
k-1 \\
r-1
\end{array}\right]_{q}-(d-1) q^{k-r-1}\left[\begin{array}{l}
k-2 \\
r-1
\end{array}\right]_{q} .
$$

Proof. Let $C, C_{1}, \ldots, C_{d}$ be $d+1$ distinct columns ( $k$-spaces) of $M$. By Lemma 4.1, $C$ contains $\left[\begin{array}{c}k \\ r\end{array}\right]_{q}$ $r$-spaces. To obtain the maximum elements in

$$
C \cap \bigcup_{i=1}^{d} C_{i}=\bigcup_{i=1}^{d}\left(C \cap C_{i}\right),
$$

we may assume that each $C_{i}$ intersects $C$ at a $(k-1)$-space. Then each $C_{i}$ covers $\left[\begin{array}{c}k-1 \\ r\end{array}\right]_{q} r$-spaces of $C$. However, the coverage of each pair of $C_{i}$ and $C_{j}$ overlaps at a $(k-2)$-space. Therefore, only $C_{1}$ covers 
the full $\left[\begin{array}{c}k-1 \\ r\end{array}\right]_{q} r$-spaces, while each of $C_{2}, \ldots, C_{d}$ can cover a maximum of $\left[\begin{array}{c}k-1 \\ r\end{array}\right]_{q}-\left[\begin{array}{c}k-2 \\ r\end{array}\right]_{q} r$-spaces not covered by $C_{1}$. Consequently, the number of $r$-spaces of $C$ not covered by $C_{1}, \ldots, C_{d}$ is at least

$$
\begin{aligned}
z & =\left[\begin{array}{l}
k \\
r
\end{array}\right]_{q}-\left[\begin{array}{c}
k-1 \\
r
\end{array}\right]_{q}-(d-1)\left(\left[\begin{array}{c}
k-1 \\
r
\end{array}\right]_{q}-\left[\begin{array}{c}
k-2 \\
r
\end{array}\right]_{q}\right) \\
& =q^{k-r}\left[\begin{array}{c}
k-1 \\
r-1
\end{array}\right]_{q}-(d-1) q^{k-r-1}\left[\begin{array}{c}
k-2 \\
r-1
\end{array}\right]_{q} .
\end{aligned}
$$

Note that for $M(m, k, r)$ to be $d^{z}$-disjunct, $z$ must be positive, which implies

$$
d<\frac{q^{k-r}\left[\begin{array}{l}
k-1 \\
r-1
\end{array}\right]_{q}}{q^{k-r-1}\left[\begin{array}{l}
k-2 \\
r-1
\end{array}\right]_{q}}+1,
$$

or $d \leq p$.

Suppose $d \leq q+1$. The following corollary shows the above $z$ is optimal.

Corollary 4.5. Suppose $k-r \geq 2$ and $1 \leq d \leq q+1$. Then $M(m, k, r)$ is not $d^{z+1}$-disjunct, where $z$ is as in Theorem 4.4.

Proof. We prove this by showing that a maximum coverage of $r$-spaces in the proof of Theorem 4.4 is obtained. We reverse the arguments. Let $U$ be a $(k-2)$-space contained in $C$. Then the number of $(k-1)$-spaces between $U$ and $C$ is

$$
\left[\begin{array}{c}
k-(k-2) \\
k-1-(k-2)
\end{array}\right]_{q}=q+1
$$

We choose $d$ distinct ones among them, say $T_{i}(1 \leq i \leq d)$. For each $T_{i}$, we choose a $k$-space $C_{i}$ such that $C \cap C_{i}=T_{i}$. Hence, each pair of $C_{i}$ and $C_{j}$ overlaps at the same $(k-2)$-space $U$.

Lemma 4.6. Suppose $r \leq \frac{k}{2}$. Then, referring to the definition of $p$ in Theorem $4.4, d=q^{r}$ is the largest integer less or equal to $p$.

Proof. Note that $q^{r-1}<q^{r} \leq q^{k-r}$. Hence

$$
\begin{aligned}
p-q^{r} & =\frac{q\left(q^{k-1}-1\right)}{q^{k-r}-1}-q^{r} \\
& =\frac{q^{k}-q-q^{k}+q^{r}}{q^{k-r}-1} \\
& =\frac{q\left(q^{r-1}-1\right)}{q^{k-r}-1} \\
& <\frac{q q^{r-1}}{q^{k-r}} \\
& \leq 1 .
\end{aligned}
$$

Then $p-1<q^{r} \leq p$. 
Corollary 4.7. Suppose $k-r \geq 2$ and $d=q^{r}$. Then $M(m, k, r)$ is $d^{z}$-disjunct with

$$
z=\left[\begin{array}{c}
k-1 \\
r-1
\end{array}\right]_{q}+\left(q^{r}-1\right)\left[\begin{array}{c}
k-2 \\
r
\end{array}\right]_{q} .
$$

Proof. Setting $d=q^{r}$ in Theorem 4.4 and referring to Lemma 4.1 and Lemma 4.3,

$$
\begin{aligned}
z & =q^{k-r}\left[\begin{array}{l}
k-1 \\
r-1
\end{array}\right]_{q}-\left(q^{r}-1\right) q^{k-r-1}\left[\begin{array}{c}
k-2 \\
r-1
\end{array}\right]_{q} \\
& =\left[\begin{array}{l}
k \\
r
\end{array}\right]_{q}-\left[\begin{array}{c}
k-1 \\
r
\end{array}\right]_{q}-\left(q^{r}-1\right)\left(\left[\begin{array}{c}
k-1 \\
r
\end{array}\right]_{q}-\left[\begin{array}{c}
k-2 \\
r
\end{array}\right]_{q}\right) \\
& =\left[\begin{array}{l}
k \\
r
\end{array}\right]_{q}-q^{r}\left[\begin{array}{c}
k-1 \\
r
\end{array}\right]_{q}+\left(q^{r}-1\right)\left[\begin{array}{c}
k-2 \\
r
\end{array}\right]_{q} \\
& =\left[\begin{array}{c}
k \\
k-r
\end{array}\right]_{q}-q^{k-(k-r)}\left[\begin{array}{c}
k-1 \\
r
\end{array}\right]_{q}+\left(q^{r}-1\right)\left[\begin{array}{c}
k-2 \\
r
\end{array}\right]_{q} \\
& =\left[\begin{array}{l}
k-1 \\
k-r
\end{array}\right]_{q}+\left(q^{r}-1\right)\left[\begin{array}{c}
k-2 \\
r
\end{array}\right]_{q} \\
& =\left[\begin{array}{l}
k-1 \\
r-1
\end{array}\right]_{q}+\left(q^{r}-1\right)\left[\begin{array}{c}
k-2 \\
r
\end{array}\right]_{q} .
\end{aligned}
$$

When $r=1$, the $z$ in Theorem 4.4 is in a neater form.

Corollary 4.8. Suppose $k \geq 3, d \leq q$, and $z=q^{k-2}(q-d+1)$. Then $M(m, k, 1)$ is $d^{z}$-disjunct, but is not $d^{z+1}$-disjunct.

Proof. Setting $r=1$ in the $z$ formula of Theorem 4.4, we obtain

$$
\begin{aligned}
z & =q^{k-1}\left[\begin{array}{c}
k-1 \\
0
\end{array}\right]_{q}-(d-1) q^{k-2}\left[\begin{array}{c}
k-2 \\
0
\end{array}\right]_{q} \\
& =q^{k-2}(q-d+1) .
\end{aligned}
$$

The second statement follows from Corollary 4.5 .

Example 4.9. Fix $q=5$. Then $M(8,4,1)$ is a $5^{25}$-disjunct matrix with 97,656 rows and 200,525,284,806 columns. This means that in our method to identify 5 positives from $2 \times 10^{11}$ clones, at most $10^{5}$ pools are necessary and 25 errors are allowed.

\section{REFERENCES}

Barrllot, E., Lacroix, B., and Cohen, D. 1995. Theoretical analysis of library screening using an $n$-dimensional pooling strategy. Nucl. Acids Res. 19, 6241-6247.

Bruno, W.J., Knill, E., Balding, D.J., Bruce, D.C., Doggett, N.A., Sawhill, W.W., Stallings, R.L., Whittaker, C.C., and Torney, E.C. 1995. Effective pooling designs of library screening. Genomics 26, 21-30.

Colburn, C., Ling, A., and Tompa, M. 2002. Construction of optimal quality control ogilio arrays. Bioinformatics 18(4), 529-535.

Du, D., and Hwang, F.K. 2000. Combinatorial Group Testing and Its Applications, 2nd ed., World Scientific, Singapore. Erdös, P., Frankl, and Füredi, D. 1985. Families of finite sets in which no set is covered by the union of $r$ others. Israel J. Math. 51, 79-89. 
Farach, et al. 1997. Group testing problems with sequences experimental molecular biology. Proc. Compression and Complexity of Sequences, 1997, IEEE Press, 357-367.

Flodman, P., Macula, A., Spence, A., and Torney, D. 2001. A new data mining technique for the analysis of simulated genetic data. Proc. Genetic Analysis Workshop 12, Wiley-Liss, 2001; Genet. Epidemiol. 21(Suppl. 1), S390-S395.

Grebinski, V., and Kucherov, G. 1998. Reconstructing a Hamiltonian cycle by querying the graph: Application to DNA physical mapping. Disc. Appl. Math. 88, 147-165.

Huang, T., and Weng, C. 2004. Pooling spaces and non-adaptive pooling designs. Disc. Math. 282(1-3), $163-169$.

Hwang, F.K. 2003. On Macula's error-correcting pool designs. Disc. Math. 267, 311-314.

Kautz, W.H., and Singleton, R.C. 1964. Nonadaptive binary superimposed codes. IEEE Trans. Inform. Theory 10, 363-377.

van Lint, J.H., and Wilson, R.M. 1992. A Course in Combinatorics, Cambridge, Victoria.

Macula, A.J. 1996a. A simple construction of $d$-disjunct matrices with certain constant weights. Disc. Math. 162, 311-312.

Macula, A.J. 1996b. Error-correcting nonadaptive group testing with $d^{e}$-disjunct matrices. Disc. Appl. Math. 80, 217222.

Macula, A.J. 1999. Probabilistic nonadaptive and two-stage group testing with relatively small pools and DNA library screening. J. Comb. Opt. 2, 385-397.

Ngo, H., and Du, D. 2002. New constructions of non-adaptive and error-tolerance pooling designs. Disc. Math. 243, 161-170.

Pevzner, P.A. 2000. Computational Molcular Biology: An Algorithmic Approach, Mass Sec. 9.5-9.6, MIT Press, Cambridge, MA.

Torney, D.C. 1999. Sets pooling designs. Ann. Combin. 3, 95-101.

Yakir, A. 1993. Inclusion matrix of $k$ vs 1 affine subspaces and a permutation module of the general affine group. J. Combin. Theory, Ser. A 63, 301-317.

Address correspondence to:

A. D'yachkov

Department of Probability Theory

Faculty of Mechanics and Mathematics

Moscow State University

Moscow, 119992, Russia

E-mail: agd-msu@yandex.ru

\title{
Author
}

Right running head okay as shown (short title)?

\author{
AU1 \\ Please provide at least three names and initial(s) for Farach.
}

